\section{Obesidad y cáncer: la tormenta perfecta}

\author{
CÉSAR SÁNCHEZ R. ${ }^{1}$, CAROLINA IBÁÑEZ ${ }^{1}$, JULIETA KLAASSEN²
}

\section{The link between obesity and cancer}

While some genetic factors may explain the development of cancer, its main causes are related to environmental exposure to carcinogenic agents as well as to the effect of determined lifestyles and habits. Several epidemiological studies have shown a consistent relation between obesity and cancer. In non smokers, obesity is the most relevant risk factor in the development of malignant tumors. There is a clear association between obesity and endometrial cancer, breast cancer in postmenopausal women, pancreatic, esophageal and colon cancer. Sexual steroids, insulin like growth factor axis and adipokines are the three main models to explain the biological basis for the obesity-cancer relationship. However, these models do not explain all the biological mechanisms that link obesity to cancer. There are other factors in play such as chronic inflammation, hypoxia and oxidative stress. Obesity may hamper the screening, diagnosis and treatment of some tumors, increasing mortality rates. Obesity prevention and management, therefore, may be the most important modifiable factor in reducing both incidence and mortality in cancer. New studies are required to quantify the effect of intentional weight reduction on the incidence and relapse of cancer. Considering the efficacy of bariatric surgery for weight reduction, it is an attractive model to study this link.

(Rev Med Chile 2014; 142: 211-221)

Key words: Bariatric surgery; Neoplasms, second primary; Obesity.

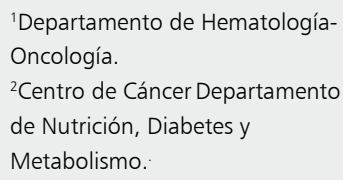

Facultad de Medicina Pontificia Universidad Católica de Chile.

Recibido el 7 de septiembre de 2012, aceptado el 7 de mayo de 2013.

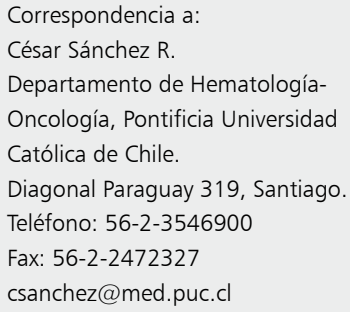

E 1 cáncer es una de las principales causas de muerte en el mundo ${ }^{1}$. En Chile es la segunda causa de mortalidad en adultos, precedida por las enfermedades cardiovasculares ${ }^{2}$. Mientras factores genéticos y hereditarios explican 5-10\% de las causas conocidas de cáncer, factores ambientales y estilos de vida dan cuenta de más del $90 \%$ restante $^{3}$. La dieta, tabaco, consumo de alcohol, infecciones, obesidad, agentes contaminantes y exposición a radiaciones influyen profundamente en el desarrollo de neoplasias, y se ha demostrado que prevenir la exposición a agentes cancerígenos ambientales tiene gran impacto en la incidencia de cáncer"; como se observa en la reducción de incidencia y mortalidad por neoplasias de la vía respiratoria-digestiva alta, luego de la disminución del hábito tabáquico (Tabla 1$)^{5}$.

En sujetos no fumadores, la obesidad, dieta y actividad física son los principales factores de riesgo (FR) asociados a neoplasias ${ }^{6}$. Se estima que $30 \%$ de la población adulta en países desarrollados es obesa, porcentaje similar a lo descrito en Chile y Estados Unidos de Norteamérica (Figuras 1A y 1B), por tanto, esta característica, en no fumadores, es el FR carcinogénico más prevalente ${ }^{7,8,9}$.

Una cohorte de pacientes con cáncer en nuestro centro muestra niveles de obesidad similares a la población general (Figura 1C).

Cambios en los estilos de vida, principalmente sedentarismo y dieta occidental, producirán un incremento de neoplasias asociadas a obesidad y de consultas de pacientes obesos con cáncer ${ }^{7}$. Es importante, por tanto, conocer los mecanismos biológicos que subyacen a esta asociación y el manejo del cáncer en obesos.

Analizaremos los mecanismos biológicos de 
Tabla 1. Causas de cáncer y potencial reducción según las medidas preventivas

\begin{tabular}{|lccc|}
\hline Agente causal & $\begin{array}{c}\text { Neoplasias relacionadas } \\
\text { (\%) }\end{array}$ & $\begin{array}{c}\text { Magnitud de la posible reducción } \\
\text { (\%) }\end{array}$ & $\begin{array}{c}\text { Tiempo* } \\
\text { (años) }\end{array}$ \\
\hline Tabaco & 33 & 75 & $10-20$ \\
Obesidad & 20 & 50 & $2-20$ \\
Virus & 5 & 100 & $20-40$ \\
Historia familiar & 5 & 50 & $2-10$ \\
Alcohol & 3 & 50 & $5-20$ \\
Radiación ionizante UV & 2 & 50 & $5-40$ \\
\hline
\end{tabular}

*Período de tiempo para lograr efecto preventivo (años). UV: ultravioleta.

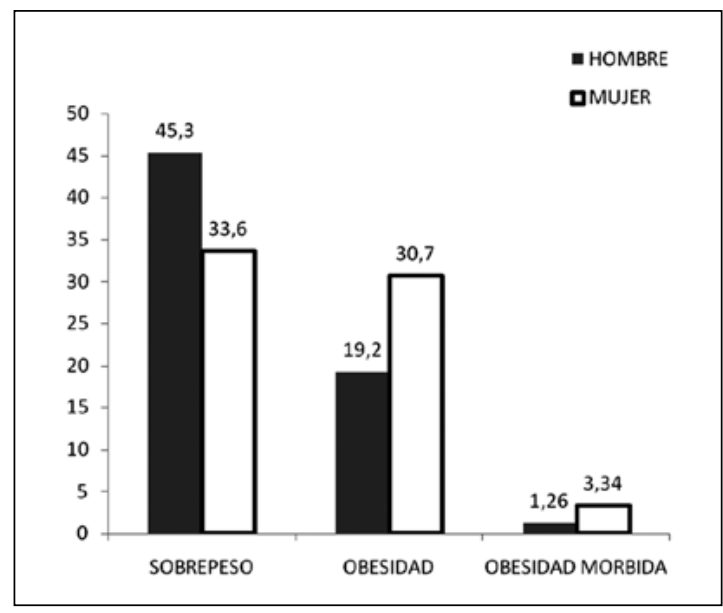

Figura 1A. Porcentaje de adultos con sobrepeso, obesidad y obesidad mórbida* en Chile el año 2010. *Definiciones según WHO (World Health Organization): Sobrepeso: IMC: 25,0$29,9 \mathrm{~kg} / \mathrm{m}^{2}$. Obesidad: IMC > $30 \mathrm{~kg} / \mathrm{m}^{2}$. Obesidad mórbida: IMC > $35 \mathrm{~kg} / \mathrm{m}^{2}$.

la relación obesidad/cáncer, el impacto de la obesidad en la prevención, diagnóstico, pronóstico y manejo de tumores sólidos.

\section{Obesidad y cáncer: evidencia epidemiológica}

La Agencia Internacional para la Investigación en Cáncer (IARC) y la World Cancer Research Fund (WCRF) sugieren que existe evidencia convincente de la relación entre obesidad y neoplasias de: Esófago (adenocarcinoma), páncreas, cáncer colo-rectal (CCR), cáncer de mama (CM) en postmenopáusicas, endometrio, renal, y probablemente cáncer de vesícula ${ }^{7}$. Basado en estas presunciones, la obesidad sería la causa subyacente

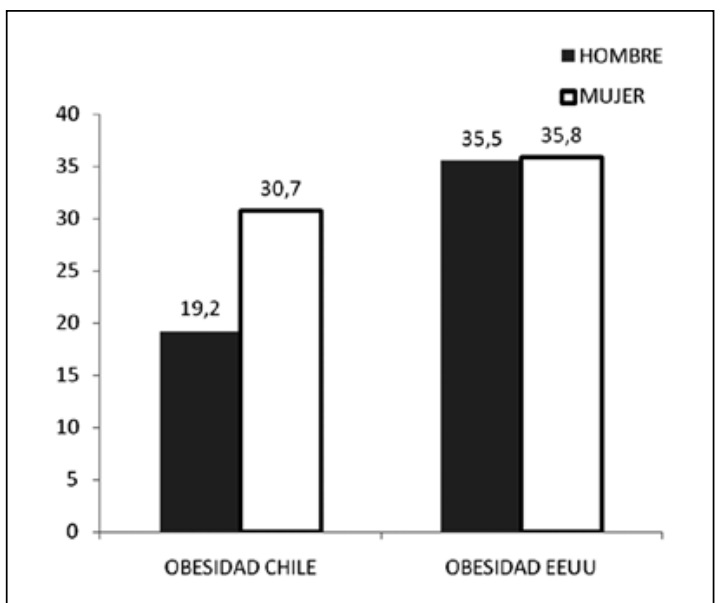

Figura 1B. Prevalencia y comparación de obesidad en Chile y Estados Unidos de Norteamérica (porcentajes). Año 2010.

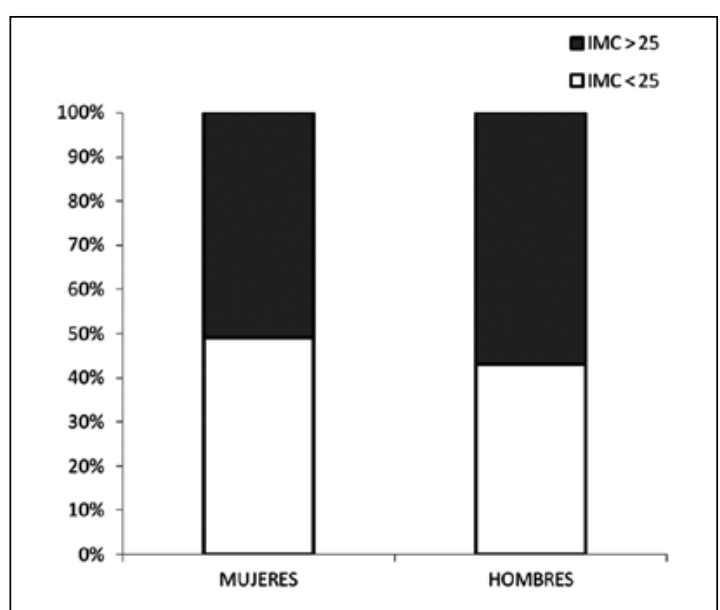

Figura 1C. Porcentaje de adultos con sobrepeso, obesidad y obesidad mórbida, con diagnóstico de cáncer en Centro de Cáncer PUC. Índice de masa corporal (IMC) de 116 pacientes adultos con cáncer al momento del diagnóstico. En esta cohorte $50,6 \%$ de las mujeres y $56,7 \%$ de los hombres con IMC $>$ 25. PUC: Pontificia Universidad Católica de Chile. 
de 39\% de los casos de cáncer endometrial, 37\% de cáncer de esófago, 25\% de cáncer renal, 11\% de CCR y 9\% de CM en mujeres postmenopáusicas $^{6}$. Esta asociación obesidad/cáncer es diferente según el estado menopáusico y género, siendo la relación obesidad-cáncer de endometrio la más fuerte y consistente (Riesgo relativo (RR): 2,5-3) $(\text { Tabla 2) })^{5,10}$.

Existe un aumento del riesgo en mujeres obesas pre-menopáusicas para CCR y melanoma maligno, y aumento en postmenopáusicas para CM y endometrio ${ }^{6,11,12}$. Así, mientras la obesidad se relaciona a $\mathrm{CM}$ en mujeres postmenopáusicas (el riesgo de CM aumenta $18 \%$ por cada $5 \mathrm{~kg} /$ $\mathrm{m}^{2}$ de ganancia de masa corporal), en pre-menopáusicas puede ser un factor protector, debido a la tendencia de estas mujeres a ciclos menstruales anovulatorios, con bajos niveles de hormonas esteroidales ${ }^{6}$. Mientras existe una asociación positiva entre índice de masa corporal (IMC) y CCR en hombres, esta asociación es más débil en mujeres; y para cáncer de recto, en donde sólo se describe en hombres ${ }^{10,13}$. Personas obesas también tienen mayor riesgo de padecer cáncer renal, tendencia más marcada en mujeres que en hombres ${ }^{10}$. La incidencia aumentada de adenocarcinomas del esófago y de cardias, en comparación con tumores escamosos en la misma localización, ha sido atribuida parcialmente a la obesidad. Lesiones precursoras de cáncer de esófago, como el esófago de Barret, se asocian a reflujo y esofagitis, ambos más habituales en obesos ${ }^{6}$. Sin embargo, el sobrepeso no explica las diferencias en la incidencia de estos tumores por sexo (el adenocarcinoma esofágico es más frecuente en hombres que en mujeres ${ }^{10}$. El estudio WHI (Women's Health Initiative) muestra que mujeres con relación cintura-cadera en el rango más alto tienen $70 \%$ de mayor riesgo de cáncer de páncreas, comparado con mujeres en rangos más bajos ${ }^{14}$.

Para neoplasias de hígado y vesícula, existe una correlación lineal entre peso y riesgo, especialmente en mujeres (RR 1,88 en mujeres vs 1,35 para hombres) ${ }^{15}$. Existiría una relación débil o nula entre obesidad y cáncer de ovario ${ }^{16,17}$.

\section{Obesidad y cáncer: factores biológicos}

Mientras la mayor parte de la evidencia que relaciona obesidad y cáncer proviene de estudios epidemiológicos como los arriba señalados, los mecanismos moleculares específicos que explican esta asociación no han sido dilucidados. Ratones con mutación en el gen de leptina, sobre-alimentados y otros, han servido como modelos preclínicos para estudiar esta relación. Estos modelos exhiben sobrecrecimiento tumoral y drogas que disminuyen el efecto metabólico de la dieta hipercalórica y obesidad (por ej; metformina), inhiben el crecimiento neoplásico en ellos ${ }^{18}$.

Denominadores comunes que definen la relación obesidad-cáncer son (Figura 2) ${ }^{19-22}$ :

1. Eje insulina/factor de crecimiento insulinosímil (IGF) tipo 1.

2. Esteroides sexuales.

3- Adiponectinas-leptina.

4. Inflamación. Citoquinas, interleukina (IL) 1, IL-6 e IL-7; factor de necrosis tumoral alfa (TNF $\alpha$ ).

Tabla 2. Aumento del riesgo de cáncer, según tumor y sexo, con incrementos de peso entre 5 y $10 \mathrm{~kg} / \mathrm{m}^{2}$

\begin{tabular}{|llll|}
\hline Tumor & $\begin{array}{l}\text { RR } \\
\text { Hombres }\end{array}$ & $\begin{array}{l}\text { RR } \\
\text { Mujeres }\end{array}$ & Mecanismo \\
Mama pre-menopáusicas & - & 0,92 & Hormonal \\
Mama postmenopáusicas & - & $1,12-1,40$ & Hormonal \\
Endometrio & - & $1,59-2,89$ & Hormonal \\
Colon & $1,24-1,45$ & $1,09-1,19$ & Insulina \\
Esófago & $1,52-2,31$ & $1,51-2,28$ & Reflujo, inflamación \\
Próstata & 1,03 & - & No claro \\
Páncreas & 1,07 & $1,12-1,25$ & Insulina \\
\hline
\end{tabular}

RR: riesgo relativo. 


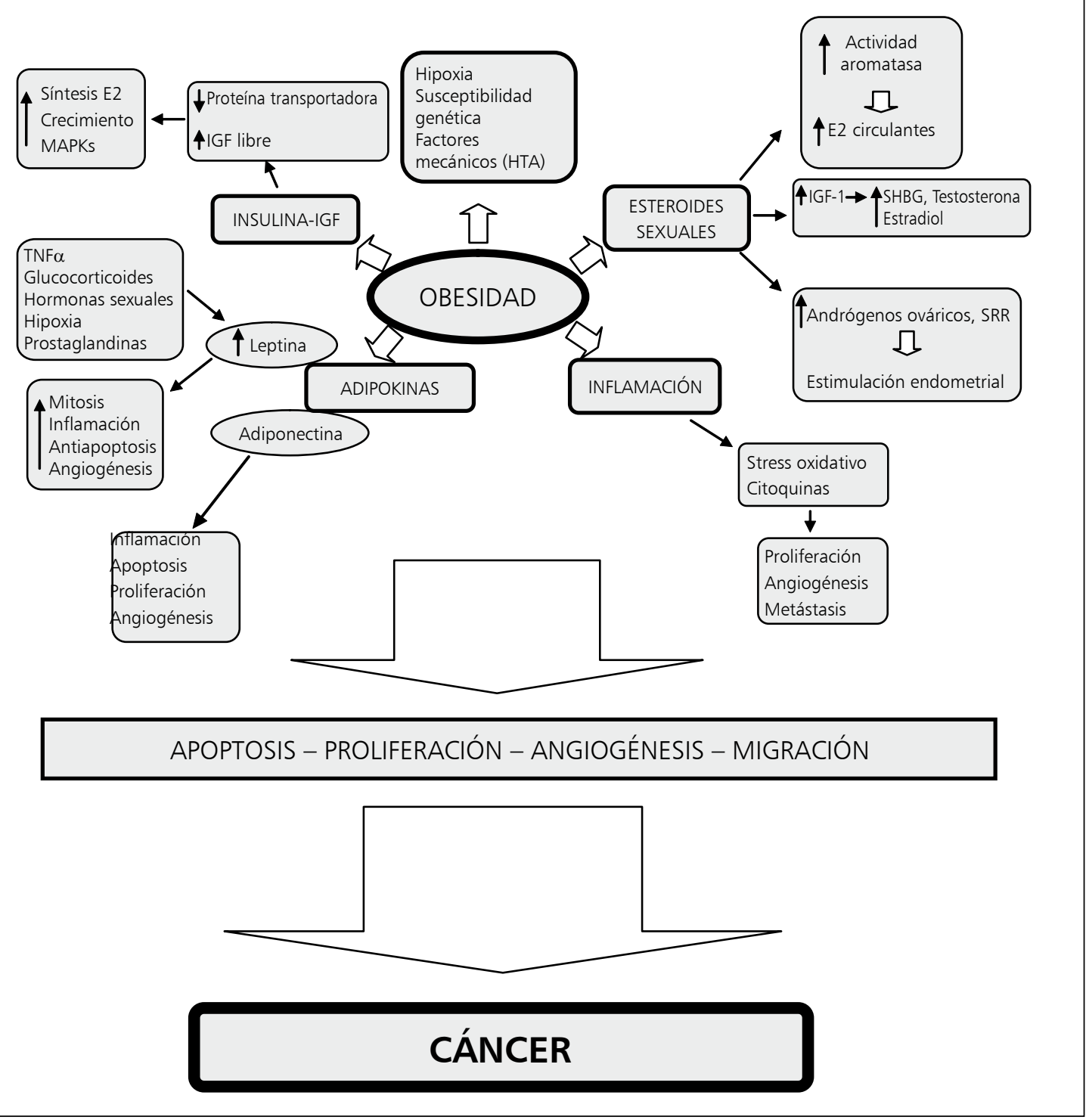

Figura 2. Mecanismos biológicos de la relación entre obesidad y cáncer. E2: Estrógenos. IGF: Factor de crecimiento insulinosímil. MAPKs: Proteínas kinasas con actividad mitogénica. TNF $\alpha$ : Factor de necrosis tumoral alfa. SHBG: Proteínas transportadoras de hormonas sexuales. SSRR: Glándulas Suprarrenales. HTA: Hipertensión arterial.

\section{Insulina-IGF}

El sobrepeso, bajos niveles de actividad física y hábitos dietéticos occidentalizados (bajo consumo de fibra, alto consumo de hidratos de carbono refinados y grasas saturadas), favorecen niveles elevados de insulina circulante ${ }^{20,22}$. La hiper-insulinemia crónica disminuye la proteína transportadora de IGF, y aumenta el IGF libre. Un sub-tipo de IGF, el IGF-1, amplifica el efecto de otros factores de crecimiento y aumenta citoquinas pro-inflamatorias y adipoquinas que regulan la síntesis de estrógenos. La insulina y también IGF-1 estimulan la actividad tirosina kinasa de receptores de vías de crecimiento, como son el receptor de insulina (IR), el receptor de IGF-1 (IGF-1R) y también el receptor híbrido de IGF-1/ 
IR, los cuales son expresados en altas cantidades en células malignas. La activación de estos receptores resulta en sobre-regulación del sustrato respondedor a insulina (insulin response substrate-1, IRS-1), el que consiguientemente activa la vía de la proteína kinasa activada por mitógenos (mitogenic-activated protein (MAP) kinase) y de la vía del fosfatidilinositide 3 kinasa/Akt (PI3KAkt) ${ }^{17,20,21}$ (Figura 3).

Existe una asociación epidemiológica entre la concentración de IGF-1 y CCR, próstata y CM en mujeres pre-menopáusicas. La concentración de IGF-1 es más alta en hombres que en mujeres lo que puede explicar las diferencias de género descritas para el CCR ${ }^{21,22}$.

\section{Esteroides sexuales}

La adiposidad influye en la síntesis y biodisponibilidad de hormonas a través de al menos tres mecanismos ${ }^{23}$.

a. Aromatasas en el tejido adiposo promueven la formación de estrógenos a partir de precursores androgénicos, siendo el tejido adiposo la principal fuente de producción de estrógenos en hombres y mujeres postmenopáusicas. Los niveles de aromatasa y niveles de estrona circulantes en mujeres postmenopáusicas están relacionados al IMC.

b. La obesidad aumenta los niveles circulantes de insulina y de IGF-1. Ambos inhiben la síntesis de globulina transportadora de hormonas sexuales (SHBG) -el principal transportador de testosterona y estradiol plasmático- y pueden llevar a un aumento en la cantidad de esteroides sexuales libres.

c. Altos niveles de insulina pueden aumentar la síntesis de andrógenos ovárica-adrenal e inducir el desarrollo del síndrome de ovario poli-quístico en pre-menopáusicas, caracterizado por hiper-androgenismo y anovulación crónica, resultante en estimulación estrogénica continua del endometrio, sin oposición de progesterona. El síndrome de ovario poli-quístico y la obesidad se asocian a un aumento del riesgo de cáncer endometrial.

\section{Adipokinas}

El tejido adiposo, especialmente visceral, es responsable de la síntesis y secreción de varios factores de crecimiento conocidos como adipokinas. De ellas, las más abundantes y estudiadas

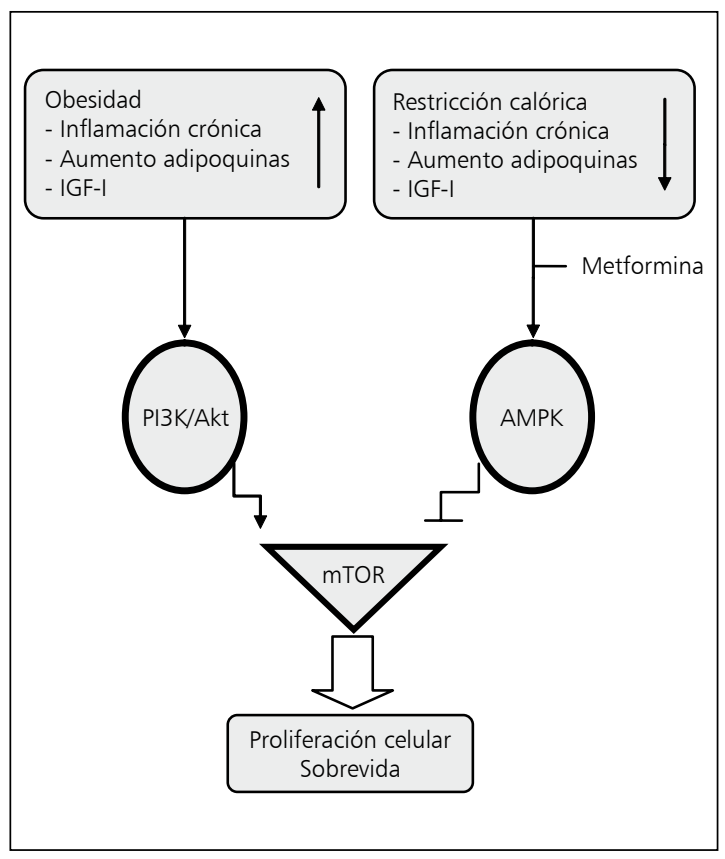

Figura 3. Metabolismo y cáncer. Mecanismos moleculares. La obesidad induce aumento de la actividad de PI3K/Akt y de mTOR estimulando la sobrevida y proliferación de células neoplásicas. Al contrario la restricción calórica y drogas como la metformina, activan la vía de AMPK inhibiendo la acción de mTOR. mTOR: mammalian target of rapamycin. AMPK: adenosine monophosphate-activated protein kinase.

son: leptina y adiponectina. Estas son mediadores claves entre el tejido adiposo, vías inflamatorias e inmunidad, y pueden influir en procesos carcinogénicos a través de una disminución (adiponectina) o un aumento (leptina) en la secreción de IL-6 y/o TNF $\alpha$ (Tabla 3$)^{23,24}$.

\section{Inflamación}

La obesidad induce un estado de inflamación y estrés oxidativo crónicos, caracterizados por la producción anómala de citoquinas, síntesis aumentada de reactantes de fase aguda y activación de señales pro-inflamatorias que contribuyen al desarrollo de insulino-resistencia, diabetes mellitus tipo 2 y ateroesclerosis relacionada a obesidad. La elevación de citoquinas es responsable de la activación de señales proliferativas, angiogénicas y metastásicas.

A pesar de una relación lógica entre estos mecanismos y la etiología de algunos tumores, existen un número de inconsistencias en este modelo ${ }^{23}$ : 
Obesidad y cáncer - C. Sánchez et al

Tabla 3. Adipoquinas, citoquinas e inflamación en la relación obesidad/cáncer

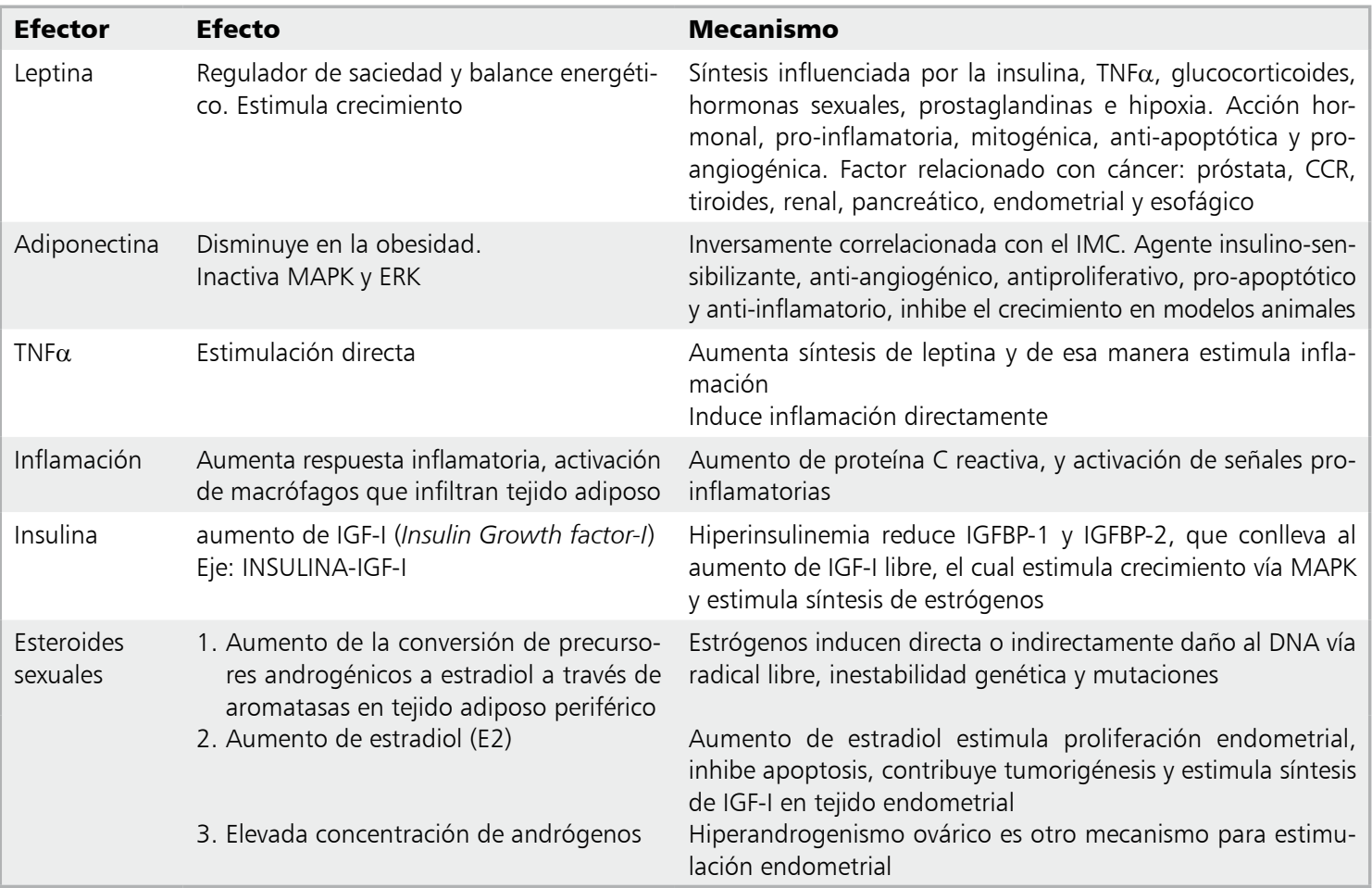

- No toma en cuenta mecanismos paracrinos, que podrían explicar la falta de relación entre niveles circulantes y tisulares de esteroides sexuales, IGF-1 y adipokinas.

- No hay relación lineal entre obesidad y niveles de IGF-1. En pacientes obesos que intencionalmente pierden peso, los niveles de IGF-1 tienden a elevarse. La complejidad del sistema insulina/IGF-1 (existen varias isoformas de re- ceptores) hace difícil su estudio y la obtención de resultados consistentes.

- Gran parte del modelo propuesto anteriormente se basa en el rol de los esteroides sexuales, y, por tanto, es sólo relevante para tumores que son sensibles a dichas hormonas; sin embargo, la obesidad también se asocia a tumores insensibles a hormonas sexuales y para ellos se han propuesto otros mecanismos (Tabla 4$)^{23}$.

\section{Tabla 4. Mecanismos biológicos que explicarían la carcinogénesis de tumores endocrino-insensibles}

\begin{tabular}{|ll|}
\hline $\begin{array}{l}\text { Mecanismo } \\
\text { Hipoxia inducida por } \\
\text { obesidad }\end{array}$ & $\begin{array}{l}\text { Descripción } \\
\text { Fuctor clave en el desarrollo de resistencia a la insulina, regulación de la inflamación crónica, } \\
\text { compartida } \\
\text { disminución de la expresión del gen de la adiponectina y aumento de los genes de las leptinas }\end{array}$ \\
$\begin{array}{l}\text { Células estromales } \\
\text { adiposas migratorias }\end{array}$ & $\begin{array}{l}\text { Células estromales y endoteliales adiposas, presentes en el tejido adiposo. Se movilizan por la } \\
\text { inflamación asociada a la obesidad y contribuyen al desarrollo tumoral }\end{array}$ \\
Factores mecánicos & $\begin{array}{l}\text { - Hipertensión: la obesidad es un FR para HTA, la que a su vez influye en el riesgo de cáncer renal } \\
\text { - Reflujo Gastro-esofágico: importante en el desarrollo de adenocarcinomas del esófago }\end{array}$ \\
& - Aumento de la absorción de iodo por la tiroides, relevante en el riesgo de cáncer tiroideo
\end{tabular}

HTA: Hipertensión arterial. 


\section{Cáncer y obesidad: factores pronósticos}

El IMC estaría relacionado a CM en etapas avanzadas, factores patológicos adversos (ausencia de receptores de estrógeno y progesterona) y menor sobrevida ${ }^{12}$. Mujeres que aumentaron su IMC $2 \mathrm{~kg} / \mathrm{m}^{2}$ tras el diagnóstico, tienen mayores probabilidades de recaída y muerte (RR: 1,64, 95\% Intervalo de Confianza; 1,07-2,51). Hunt y Sickles ${ }^{25}$, además, describen un leve aumento de CM de intervalo (aquel que aparece entre exámenes de screening).

Mujeres obesas tienen mayor mortalidad por cáncer de cuello uterino y hombres obesos tienen con alta frecuencia tumores prostáticos de gran volumen, alta frecuencia de márgenes positivos $\mathrm{y}$ factores patológicos adversos ${ }^{26}$. El IMC sería un predictor independiente de falla bioquímica tras prostatectomía y $\mathrm{RT}^{27}$.

La obesidad no sólo aumentaría la incidencia de neoplasias hepáticas primarias sino que también incrementaría su mortalidad ${ }^{28}$. Mientras confiere mejor sobrevida específica en pacientes con cáncer renal localizado ${ }^{29}$, su influencia pronóstica en cáncer de ovario y de vejiga no es consistente ${ }^{16,30}$.

\section{Obesidad y cáncer: dificultades diagnósticas}

Es menos frecuente que mujeres obesas participen en programas de detección precoz de $\mathrm{CM}$ y cérvico-uterino $^{31,32}$. Las causas serían: 1) dificultad técnica en el examen físico; 2) equipamiento inadecuado y 3) negación y rechazo por el paciente. El temor y vergüenza a desvestirse y evitar comentarios negativos en relación al peso son las barreras más frecuentemente esgrimidas en mujeres extremadamente obesas. Educar o motivar a pacientes para manejar sus temores y a los médicos acerca de técnicas específicas del examen físico, adecuado equipamiento y entrenamiento en el manejo de temores y barreras impuestas por las pacientes, podrían aumentar los niveles de tamizaje $\mathrm{e}^{32,33}$.

En programas de tamizaje de otros tumores, como CCR, la obesidad parece no jugar un $\mathrm{rol}^{34,35}$.

\section{Manejo del cáncer en el paciente obeso}

El 14 a 20\% de las muertes por cáncer en adultos mayores de 50 años son atribuibles al sobrepeso y obesidad, y se estima que más de 90.000 muertes anuales por cáncer se evitarían si la población adulta mantuviera un IMC bajo 25 durante toda su vida ${ }^{3}$ (Tabla 5A).

El escenario de obesidad y cáncer genera 2 preguntas:

1. ¿Cómo tratar un paciente obeso con cáncer?

2. ¿Tiene la reducción programada de peso efectos sobre la incidencia y el pronóstico del cáncer? Intentaremos responder, brevemente, a estas preguntas.

\section{Tratamiento oncológico en el paciente obeso con cáncer}

\section{Cirugía}

Pacientes obesos tienen mayores tasas de complicaciones post-operatorias como: eventos trombo-embólicos (RR: 1,5) ${ }^{36}$, cicatrización lenta, tiempos operatorios y estadías hospitalarias prolongadas $^{12}$. La obesidad puede ser un factor limitante en la etapificación, estudio endoscópico y disección ganglionar en tumores de cabeza y cuello $^{37}$ y en la etapificación quirúrgica del cáncer endometrial ${ }^{38}$; en este y en tumores renales la cirugía laparoscópica ha demostrado menos complicaciones hemorrágicas, mejor etapificación y menores estadías hospitalarias ${ }^{39,40}$. La cistectomía tiene mayores desafíos técnicos y riesgos posoperatorios $^{40}$. Pacientes obesos sometidos a cirugía por cáncer rectal tras neoadyuvancia, tienen estadías hospitalarias y tiempos quirúrgicos más largos, sin comprometer los resultados oncológicos en manos de cirujanos expertos ${ }^{41}$.

Kayani mostró en un meta-análisis que pacientes obesos, no diabéticos, sometidos a esofagectomía no tenían mayores complicaciones ${ }^{42}$.

\section{Radioterapia}

La planificación de tratamientos en pacientes con alto IMC requiere más dedicación, para prevenir errores sistemáticos en las direcciones laterales y longitudinales, los que de no corregirse diariamente pueden resultar en una entrega inadecuada de radiación sobre el tumor y órganos colindantes. Estos ajustes han demostrado ser relevantes en cánceres de próstata, recto, endometrio y cervicouterino ${ }^{43}$. Cinco estudios de radioterapia externa han sugerido que los resultados de la radioterapia en cáncer de próstata en pacientes obesos son inferiores. Análisis retrospectivos de pacientes 
Obesidad y cáncer - C. Sánchez et al

tratadas por cáncer de endometrio mostraron que la mortalidad por cáncer a 4 años fue $10 \%$ mayor en obesas ${ }^{43,44}$.

\section{Quimioterapia (QT)}

Habitualmente las dosis de QT se ajustan según el área de superficie corporal (SC). Para esquemas de QT habitualmente usados en el tratamiento de CCR, pulmón, ginecológicos o CM, no se ha demostrado mayor toxicidad cuando la dosis es calculada en base a la SC real, incluso si esta es mayor a 2; sin embargo, debido al temor a toxicidades no deseadas, es frecuente el cálculo de dosis según "peso ideal” o hasta un máximo de SC de 2,245-47. La reducción de dosis podría comprometer la eficacia del tratamiento. Un análisis retrospectivo del Cancer and Leukemia Group B 8541, muestra que mujeres obesas con CM en etapa precoz, y que reciben QT en dosis según la $\mathrm{SC}$, no experimentan mayor toxicidad, mientras que aquellas que reciben dosis reducidas tiene una peor sobrevida libre de enfermedad ${ }^{48}$; así, el peor pronóstico de CM en mujeres obesas, podría explicarse por el sub-tratamiento. Recientemente, la Sociedad Americana de Oncología Clínica (ASCO) ha sugerido el uso de la SC real para el cálculo de la mayoría de las QT en pacientes con IMC elevado ${ }^{49}$.

\section{Reducción de peso, cáncer y obesidad}

El reporte del WCRF del año $2007^{50}$ evaluó el impacto de la actividad física y nutrición en la prevención del cáncer, y concluyó que existe evidencia emergente de que aspectos de la nutrición y actividad física, a través del control de peso, pueden ayudar a disminuir su recurrencia. Una actualización sugiere además, que una dieta baja en grasa y alta en fibra puede evitar la recaída y progresión del CM, CCR y prostático ${ }^{51,52}$. La mayoría de estas conclusiones se basan en estudios observacionales ${ }^{53}$. Estudios prospectivos indican que la actividad física periódica, mejora el pronóstico de CM, de próstata y CCR; describiéndose una relación dosis respuesta entre actividad física y pronóstico ${ }^{53}$.

Patterson et al, basado en 2 estudios, el WINS (Women Intervention Nutrition Study) y el WHEL (Women's Healthy Eating and Living), concluye que la baja de peso, y no la dieta por sí sola, es la causante de la reducción en la incidencia de $\mathrm{CM}^{54}$. La pérdida de peso también está asociada con bajo
Tabla 5. Recomendaciones nutricionales y cáncer

\section{A. Prevención. Estrategias de prevención de cáncer y nutrición}

Mantener peso saludable
Consumir $\geq 5$ frutas, vegetales/día
Disminuir hidratos de carbono refinados
Disminuir consumo de carnes rojas
Limitar consumo de alcohol
Actividad física moderada por 30 min $\geq 5$ veces semanales

B. Tratamiento. Tratamiento de la obesidad en pacientes con cáncer

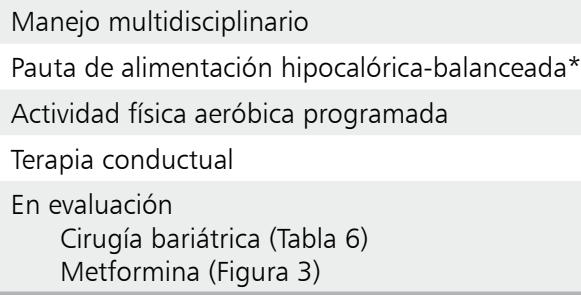

*Aporte adecuado de macro-nutrientes, restricción calórica individualizada.

riesgo de reflujo gastro-esofágico, un FR plausible para el desarrollo de adenocarcinoma del esófago ${ }^{6}$.

La mantención de un peso saludable a lo largo de la vida disminuye la incidencia de patologías neoplásicas y mejora la evolución de estas. Dado que muchos estudios clínicos aleatorios controlados (RCT) muestran que perder $5-10 \%$ de peso impacta favorablemente el riesgo de otras co-morbilidades (diabetes, hipertensión, dislipidemias) ${ }^{55}$, guías actuales recomiendan evitar ganancias de peso tras el diagnóstico de cáncer ${ }^{51,56}$ (Tabla 5B).

Sin embargo, a nivel poblacional y en estudios RCT, la evidencia de que reducir los niveles de obesidad, intencionalmente, lleve a una disminución en la incidencia de cáncer es limitada ${ }^{51}$, probablemente porque no existen estrategias efectivas para reducir el sobrepeso, masiva y sostenidamente; $y$ es difícil separar en estos estudios, los efectos de la actividad física, la dieta y su evolución a largo plazo.

\section{Cirugía bariátrica y reducción del riesgo de cáncer}

La mayoría de las intervenciones médicas que modifican estilos de vida logran pérdidas de peso de entre 7-10\%. La cirugía bariátrica $(\mathrm{CB})$ resulta 
Tabla 6. Cirugía bariátrica y cáncer. Resultados de algunos estudios clínicos

\begin{tabular}{|c|c|c|c|c|c|c|}
\hline $\begin{array}{l}\text { Autor/ } \\
\text { año }\end{array}$ & $\begin{array}{c}\text { Grupo } \\
\text { cirugía } \\
\text { (n) }\end{array}$ & $\begin{array}{l}\text { Grupo } \\
\text { control } \\
\text { (n) }\end{array}$ & Objetivo evaluado & 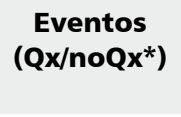 & Riesgo & $\begin{array}{l}\text { Seguimiento } \\
\text { (años) }\end{array}$ \\
\hline $\begin{array}{l}\text { Adams/ } \\
2009\end{array}$ & 6.596 & 9.442 & $\begin{array}{l}\text { Incidencia de cáncer } \\
\text { Mortalidad por cáncer }\end{array}$ & $\begin{array}{c}254 / 477 \\
41 / 107\end{array}$ & $\begin{array}{ll}H R=0,76 & P=0,0006 \\
H R & =0,54 \quad P=0,001\end{array}$ & 12,3 \\
\hline $\begin{array}{l}\text { Sjöström/ } \\
2009\end{array}$ & 2.020 & 2.037 & Incidencia cáncer & $117 / 169$ & $H R=0,67 \quad P=0,009$ & 10,9 \\
\hline $\begin{array}{l}\text { Christou/ } \\
2008\end{array}$ & 1.035 & 5.746 & $\begin{array}{l}\text { Visitas relacionadas a } \\
\text { cáncer }\end{array}$ & $21 / 487$ & $R R=22,9 \quad P=0,001$ & 5 \\
\hline
\end{tabular}

${ }^{*}$ Qx/no Qx: grupo cirugía/grupo control. n: Número de pacientes. HR: Hazard ratio. RR: Riesgo relativo.

en pérdidas de 30\%-50\% al año y obesos sometidos a esta cirugía parecen tener bajos niveles de neoplasias $^{57-62}$ (Tabla 6). Christou y cols muestran que obesos sometidos a $\mathrm{CB}$ tienen menos visitas médicas por diagnóstico de cáncer que controles $(2 \% \text { versus } 8,5 \%, R R \text { de } 0,22, p=0,001)^{60}$. Adams y cols compararon la incidencia y mortalidad por cáncer en pacientes sometidos a CB y obesos controles. La incidencia de neoplasias en el grupo quirúrgico fue menor que en el grupo control ${ }^{61}$. Esto principalmente debido a una disminución de cánceres diagnosticados en etapas avanzadas; especialmente en mujeres y en neoplasias relacionadas a obesidad. Mientras el beneficio en incidencia fue sólo para tumores relacionados a obesidad, la diminución de la mortalidad por cáncer fue observada también en neoplasias no relacionadas. Sjöström, en un estudio prospectivo, muestra que la $\mathrm{CB}$ reduce el riesgo de desarrollar cáncer, principalmente en mujeres (RR 0,58, 95\% CI 0,44-0,77). La mortalidad ajustada fue $30,7 \%$ más baja en el grupo quirúrgico $(p=0,0102)$ y menos personas murieron por cáncer en este grupo (47 en controles y 29 en grupo de cirugía $)^{62}$.

Mientras la CB puede ser una estrategia efectiva para la prevención de cáncer, aun no ha sido analizado su impacto sobre las recurrencias.

\section{Conclusiones}

Mientras la asociación epidemiológica obesidad/cáncer es evidente y algunos estudios prospectivos sustentan una relación causa-efecto, sus bases biológicas no son totalmente comprendidas. $\mathrm{Si}$ bien la reducción de peso, en no fumadores, es "el factor modificable" más importante sobre el riesgo de cáncer, no sabemos cuánto peso es necesario perder para reducir este riesgo, qué latencia existe para el desarrollo del beneficio y cómo influye sobre el riesgo la recuperación de peso ${ }^{53}$.

La prevalencia de obesidad ha aumentado considerablemente en los últimos años, a nivel nacional como internacional. El enfoque de prevención y tratamiento de ella no sólo debiera focalizarse en las enfermedades crónicas no transmisibles, sino que también debiera considerar patologías oncológicas como las mencionadas anteriormente.

\section{Referencias}

1. http://info.cancerresearchuk.org/cancerstats/world/theglobal-picture/. (Fecha de acceso 28 de agosto de 2012).

2. http://www.ine.cl/canales/chile_estadistico/demogra fia_y_vitales/estadisticas_vitales/2011/Vitales_2009_ 21102011.pdf. (Fecha de acceso 28 de agosto de 2012).

3. Anand P, Kunnumakkara AB, Sundaram C, Harikumar $\mathrm{KB}$, Tharakan ST, Lai OS, et al. Cancer is a preventable disease that requires major lifestyle changes. Pharm Res 2008; 25: 2097-116.

4. Eheman C, Henley SJ, Ballard-Barbash R, Jacobs EJ, Schymura MJ, Noone AM, et al. Annual Report to the Nation on the status of cancer, 1975-2008, featuring cancers associated with excess weight and lack of sufficient physical activity. Cancer 2012; 118: 2338-66.

5. Colditz GA, Wolin KY, Gehlert S. Applying What We Know to Accelerate Cancer Prevention. Sci Transl Med 2012; 4: 1-9.

6. Wolin KY, Carson K, Colditz GA. Obesity and cancer. Oncologist 2010; 15: 556-65.

7. http://www.dietandcancerreport.org/cancer_resouree_center/er_full_report_english.php. (Fecha de acceso 28 de agosto de 2012). 
8. Encuesta Nacional de Salud ENS 2009-2010. www.redsalud.gov.cl/(Fecha de acceso 28 de agosto de 2012).

9. http://www.cdc.gov/obesity/data/adult.html. (Fecha de acceso 28 de agosto de 2012).

10. Key TJ, Spencer EA, Reeves GK. Symposium 1: Overnutrition: consequences and solutions. Obesity and cancer risk. Proc Nutr Soc 2010; 69: 86-90.

11. Macciò A, Madeddu C. Obesity, inflammation, and postmenopausal breast cancer: therapeutic implications. Scientific World Journal 2011; 11: 2020-36.

12. Haakinson DJ, Leeds SG, Dueck AC, Gray RJ, Wasif N, Stucky CC, et al. The Impact of Obesity on Breast Cancer: A Retrospective Review. Ann Surg Oncol 2012 Mar 27. [Epub ahead of print].

13. Rosen $\mathrm{AB}, \mathrm{Schneider} \mathrm{EC}$. Colorectal cancer screening disparities related to obesity and gender. J Gen Intern Med 2004; 19: 332-8.

14. Luo J, Horn K, Ockene JK, Simon MS, Stefanick ML, Tong E, et al. Interaction between smoking and obesity and the risk of developing breast cancer among postmenopausal women: the Women's Health Initiative Observational Study. Am J Epidemiol 2011; 174: 91928.

15. Larsson SC, Wolk A. Obesity and the risk of gallbladder cancer: a meta-analysis. Br J Cancer 2007; 96: 1457-61.

16. Kuper H, Cramer DW, Titus-Ernstoff L. Risk of ovarian cancer in the United States in relation to anthropometric measures: does the association depend on menopausal status? Cancer Causes Control 2002; 13: 455-63.

17. Leitzmann MF, Koebnick C, Danforth KN, Brinton LA, Moore SC, Hollenbeck AR, et al. Body mass index and risk of ovarian cancer. Cancer 2009; 115: 812-22.

18. Kanasaki K, Koya D. Biology of Obesity: Lessons from Animal Models of Obesity. J Biomed Biotechnol. vol. 2011, Article ID 197636, 11 pages, 2011. doi:10.1155/2011/197636.

19. The Role of Obesity in Cancer Survival and Recurrence: Workshop Summary http://www.nap.edu/catalog. php?record_id=13348 (Fecha de acceso 28 de agosto de 2012).

20. Gallagher EJ, Leroith D. Insulin, insulin resistance, obesity, and cancer. Curr Diab Rep 2010; 10: 93-100.

21. Chen J. Multiple signal pathways in obesity-associated cancer. Obes Rev 2011; 12: 1063-70.

22. Pollak M. The insulin and insulin-like growth factor receptor family in neoplasia: an update. Nat Rev Cancer 2012; 12: 159-69.

23. Roberts DL, Dive C, Renehan AG. Biological mechanisms linking obesity and cancer risk: new perspectives. Annu Rev Med 2010; 61: 301-16.

24. Karastergiou K, Mohamed-Ali V. The autocrine and paracrine roles of adipokines. Mol Cell Endocrinol 2010; 318: 69-78.

25. Hunt KA, Sickles EA. Effect of obesity on screening mammography: outcomes analysis of 88,346 consecutive examinations. AJR Am J Roentgenol 2000; 174: 1251-5.

26. Buschemeyer WC 3rd, Freedland SJ. Obesity and prostate cancer: epidemiology and clinical implications. Eur Urol 2007; 52: 331-43.

27. Strom SS, Kamat AM, Gruschkus SK, Gu Y, Wen S, Cheung MR, et al. Influence of obesity on biochemical and clinical failure after external-beam radiotherapy for localized prostate cancer. Cancer 2006; 107: 631-9.

28. Tanaka K, Tsuji I, Tamakoshi A, Matsuo K, Ito H, Wakai $\mathrm{K}$, et al. Research Group for the Development and Evaluation of Cancer Prevention Strategies in Japan. Obesity and liver cancer risk: an evaluation based on a systematic review of epidemiologic evidence among the Japanese population. Jpn J Clin Oncol 2012; 42: 212-21.

29. Rogde AJ, Gudbrandsdottir G, Hjelle KM, Sand KE, Bostad L, Beisland C. Obesity is associated with an improved cancer-specific survival, but an increased rate of postoperative complications after surgery for renal cell carcinoma. Scand J Urol Nephrol 2012 Apr 25. [Epub ahead of print].

30. Hafron J, Mitra N, Dalbagni G, Bochner B, Herr H, Donat SM. Does body mass index affect survival of patients undergoing radical or partial cystectomy for bladder cancer? J Urol 2005; 173: 1513-7.

31. Maruthur NM, Bolen S, Brancati FL, Clark JM. Obesity and mammography: a systematic review and metaanalysis. J Gen Intern Med 2009; 24: 665-77.

32. Wee CC, Mccarthy EP, Davis RB, Phillips RS. Screening for cervical and breast cancer: is obesity an unrecognized barrier to preventive care? Ann Intern Med 2000; 132: 697-704.

33. Mitchell RS, Padwal RS, Chuck AW, Klarenbach SW. Cancer screening among the overweight and obese in Canada. Am J Prev Med 2008; 35: 127-32.

34. Skolarus TA, Wolin KY, Grubb RL 3rd. The effect of body mass index on PSA levels and the development, screening and treatment of prostate cancer. Nat Clin Pract Urol 2007; 4: 605-14.

35. Fagan HB, Myers RE, Daskalakis C, Sifri R, Mainous AG 3rd, Wender R. Race/Ethnicity, gender, weight status, and colorectal cancer screening. J Obes 2011; 2011: 314619.

36. De Martino RR, Goodney PP, Spangler EL, Wallaert JB, Corriere MA, Rzucidlo EM, et al. Variation in thromboembolic complications among patients undergoing commonly performed cancer operations. J Vasc Surg 2012; 55: 1035-40. 
37. Deneuve S, Tan HK, Eghiaian A, Temam S. Management and outcome of head and neck squamous cell carcinomas in obese patients. Oral Oncol 2011; 47: 631-5.

38. Hauspy J, Jiménez W, Rosen B, Gotlieb WH, Fung-KeeFung M, Plante M. Laparoscopic surgery for endometrial cancer: a review. J Obstet Gynaecol Can 2010; 32: 570-9.

39. Acholonu Uc JR, Chang-Jackson SC, Radjabi AR, Nezhat FR. Laparoscopy for the management of early-stage endometrial cancer: from experimental to standard of care. J Minim Invasive Gynecol 2012; 19: 434-42.

40. Stewart SB, Freedland SJ. Influence of obesity on the incidence and treatment of genitourinary malignancies. Urol Oncol 2011; 29: 476-86.

41. Chern H, Chou J, Donkor C, Shia J, Guillem JG, Nash GM, et al. Effects of obesity in rectal cancer surgery. J Am Coll Surg 2010; 211: 55-60.

42. Kayani B, Okabayashi K, Ashrafian H, Harling L, Rao C, Darzi A, et al. Does Obesity Affect Outcomes in Patients Undergoing Esophagectomy for Cancer? A Meta-analysis. World J Surg 2012; 36: 1785-95.

43. Lin LL, Hertan L, Rengan R, Teo BK. Effect of body mass index on magnitude of setup errors in patients treated with adjuvant radiotherapy for endometrial cancer with daily image guidance. Int J Radiat Oncol Biol Phys 2012; 83: 670-5.

44. Martra F, Kunos C, Gibbons H, Zola P, Galletto L, Debernardo R, et al. Adjuvant treatment and survival in obese women with endometrial cancer: an international collaborative study. Am J Obstet Gynecol 2008; 198: 89.e1-8.

45. Lopes-Serrao MD, Ussery SM, Hall RG 2nd, Shah SR. Evaluation of chemotherapy-induced severe myelosuppression incidence in obese patients with capped dosing. J Oncol Pract 2011; 7: 13-7.

46. Field KM, Kosmider S, Jefford M, Michael M, Jennens R, Green $\mathrm{M}$, et al. Chemotherapy dosing strategies in the obese, elderly, and thin patient: results of a nationwide survey. J Oncol Pract 2008; 4: 108-13.

47. Griggs JJ, Sorbero ME, Lyman GH. Undertreatment of obese women receiving breast cancer chemotherapy. Arch Intern Med 2005; 165: 1267-73.

48. Rosner GL, Hargis JB, Hollis DR, Budman DR, Weiss RB, Henderson IC, et al. Relationship between toxicity and obesity in women receiving adjuvant chemotherapy for breast cancer: results from cancer and leukemia group B study 8541. J Clin Oncol 1996; 14: 3000-8.

49. Griggs JJ, Mangu PB, Anderson H, Balaban EP, Dignam JJ, Hryniuk WM, et al. American Society of Clinical Oncology. Appropriate chemotherapy dosing for obese adult patients with cancer: American Society of Clinical
Oncology clinical practice guideline. J Clin Oncol 2012; 30: 1553-61.

50. Bekkering T, Beynon R, Davey Smith G, Davies A, Harbord R, Sterne J, et al. 2006. A systematic review of RCTs investigating the effect of dietal and physical activity interventions on cancer survival, updated report World Cancer Research Fund, http://www.dietandcancerreport. org/(Fecha de acceso 28 de agosto de 2012).

51. Davies NJ, Batehup L, Thomas R. The role of diet and physical activity in breast, colorectal and prostate cancer survivorship: a review of the literature. Br J Cancer 2011; 105 Suppl 1: S52-73.

52. Pekmezi DW, Demark-Wahnefried W. Updated evidence in support of diet and exercise interventions in cancer survivors. Acta Oncol 2011; 50: 167-78.

53. Byers T, Sedjo RL. Does intentional weight loss reduce cancer risk? Diabetes Obes Metab 2011; 13: 1063-72.

54. Patterson RE, Cadmus LA, Emond JA, Pierce JP. Physical activity, diet, adiposity and female breast cancer prognosis: a review of the epidemiologic literature. Maturitas 2010; 66: 5-15.

55. Knowler WC, Barrett-Connor E, Fowler SE, Hamman RF, Lachin JM, Walker EA, et al. Diabetes Prevention Program Research Group. Reduction in the incidence of type 2 diabetes with lifestyle intervention or metformin. N Engl J Med 2002; 346: 393-403.

56. NHLBI Obesity Education Initiative Expert panel on the Identification, 1998. http://www.ncbi.nlm.nih.gov/ books/NBK2003/pdf/TOC.pdf. (Fecha de acceso 28 de agosto de 2012).

57. http://www.cancer.gov/cancertopics/factsheet/Risk/ obesity (Fecha de acceso 28 de agosto de 2012).

58. Anderson AS, Caswell S. Obesity management-an opportunity for cancer prevention. Surgeon 2009; 7: 282-5.

59. Chang SH, Stoll CR, Colditz GA. Cost-effectiveness of bariatric surgery: Should it be universally available? Maturitas 2011; 69: 230-8.

60. Christou NV, Lieberman M, Sampalis F, Sampalis JS. Bariatric surgery reduces cancer risk in morbidly obese patients. Surg Obes Relat Dis 2008; 4: 691-5.

61. Adams TD, Stroup AM, Gress RE, Adams KF, Calle EE, Smith SC, et al. Cancer incidence and mortality after gastric bypass surgery. Obesity (Silver Spring) 2009; 17: 796-802.

62. Sjöström L, Gummesson A, Sjöström CD, Narbro K, Peltonen M, Wedel H, et al. Swedish Obese Subjects Study. Effects of bariatric surgery on cancer incidence in obese patients in Sweden (Swedish Obese Subjects Study): a prospective, controlled intervention trial. Lancet Oncol 2009; 10: 653-62. 